\title{
ADA AND EXPERT SYSTEMS: THE INTELLIGENT MISSILE PROJECT
}

\author{
Thomas Mossberg
}

Code 3922

Naval Weapons Center

China Lake, CA 93555-6001

Like most other weapon systems, missile are now required to perform with less human intervention and increased reliability. The use of artificial intelligence (AI) technology, which gives missiles some decision-making capability, is one way to achieve these performance requirements. The Intelligent Missile Project was formed to develop techniques for embedding rule-based AI systems in the U.S. Navy's tactical air-to-air and air-to-surface missiles, and to concurrently develop tools that will assist in AI systems development. This paper discusses the overall approach taken by the project, including some of the technical problems encountered, solutions to these problems, and two missile applications where AI technology is being used.

\section{Background}

The Intelligent Missile Project was initiated to develop and demonstrate advanced software techniques that can provide increased missile autonomy and reliability and reduced life-cycle costs. Like aircraft, missiles face an increasingly hostile threat environment. The demand for fire-and-forget missiles is on the upswing. Such missiles are pointed and fired in the general direction of the intended target. Then, while the delivery platform continues to the next objective (clearing the area or looking for the next target), the missile picks out and destroys the desired target. This type of autonomous operation requires a degree of decision making not found in currently available missiles. To be useful, such advanced weapons must be both dependable and affordable.

Both AI and software engineering technologies are being used to meet reliability and affordability requirements. In an evolutionary effort, the Intelligent Missile Project is using AI to supplement and complement current algorithmic techniques. AI technology provides a different way of viewing problems, and offers suggestions for new approaches. AI also allows a method for easily incorporating knowledge into software. The software can then infer decisions.

The Intelligent Missile Project was started to achieve the potential payoffs of using AI techniques. Project personnel are to develop tasked techniques for embedding rule-based AI systems in the U.S. Navy's tactical air-to-air and air-to-surface missiles. The Project's objective is to demonstrate the techniques and tools necessary to achieve the level of autonomous performance required by advanced weapons. At the same time, the Project will address issues concerned with the life-cycle costs of such weapon systems.

\section{Approach}

Because of the desired time frame for acquiring these missile performance capabilities, we are constrained to using a level of computer resources consistent with the next generation of missile computers because they are faster and cheaper than the dedicated military hardware. While computer memory is not a severely constrained as it has been in the past, we still must exercise some restraint because the amount of memory available runs from 512 KBytes for a large tactical missile to $48 \mathrm{KBytes}$ for a small one.

The Project's four main tasks take into account the environment in which the software must operate. One task examines different types of rule-based systems in light of their applicability and efficiency. A second task involves looking at the tools that are needed to develop software that combines rule-based systems with algorithmic and control software. A third task explores hardware assistance for rule-based and Ada systems. The last task is concerned with applying the developed techniques to actual missiles.

This approach allows some of the tasks to proceed in parallel. Information learned while developing different rule-based systems is used in determining what capabilities are needed by the tools. The use of the developed techniques with definite applications guides the exploration of rule-based system technology and keeps it from becoming just an academic exercise. The application of this technology to more than one missile system will help in assessing the validity of using rule-based systems.

\section{Expert Systems}

A missile's operating environment makes the development of rule-based systems simultaneously both easier and harder. No human in the loop makes development easier because there is no human interface to worry about. However, no human in the loop makes development harder for the very same reason. If the system gets stuck or encounters a situation not covered by the rule set, there is literally nobody it can ask for help. 
Additionally, the missile's operational timing constraints impose an execution constraint not found in a typical expert system. Most of the expert systems found in use have human response-time requirements that are measured on the order of seconds. A missile's frame times range from one second to .5 milliseconds. The requisite performance of a rule-based system will depend upon what part of the missile system it is assisting.

As mentioned previously, we have to be somewhat frugal in our memory usage. Several commercial expert shells that allow embedding of their reasoning functions in an application were examined. These shells require a memory capacity on the order of $100 \mathrm{KBytes}$ or more, which was not acceptable for tactical missile applications. However, for designing, developing and validating rules, the shells performed reasonably well. This examination lead us to concentrate the initial effort on designing and implementing various types of rule-based systems with an emphasis on execution efficiency. We did not develop any software for entering, editing, and debugging rule sets. Instead, we relied on commercially available tools to perform these functions.

We've already had experience in using Ada successfully for implementing guidance and control algorithms for missiles. Here was an opportunity to push the envelope and learn how Ada performed in AI applications. While LISP has its strengths, using a single language for both rule-based and numeric/control software is a definite cost advantage.

The first rule-based system that was developed was a simple expert system that could do forward and backward chaining. In forward chaining, the inference engine (the software that does the reasoning) starts with a set of known conditions and then deduces new conclusions. Backward chaining works in the opposite manner given a set of conclusions, the inference engine infers what the starting conditions must be. We use the term "conditions" as opposed to "facts" because the word facts implies something that does not change, while the word conditions implies mutability over time. The data structures that allow this are shown in Figure 1. The conditions record is stored in an AVL Tree while the other records are stored using linked lists. As a condition is asserted, it is put on a list. When the inference engine is asked to draw conclusions, it takes a condition off the list and finds the first rule where that condition is used as an antecedent. The inference engine then checks to see if all of the rule's antecedents have been asserted, and if they have, then the inference engine puts all of that rule's consequences on a list containing the conclusions and at the end of the antecedent list. The inference engine then traverses the antecedent list until that list is empty. In this fashion, the inference engine checks only those rules for which it knows that at least one antecedent is true, keeping rule checking to a minimum. The inference engine then finds all possible conclusions, handling the case where a consequence is used as an antecedent.

The second rule-based system developed was a decision tree. When work on this system started, none of the available expert shells could generate a decision tree in Ada. This situation necessitated building our own simple generator, again leaving the front end to the existing tools. Our tool builds an optimized decision tree, so that only the minimum number of antecedents must be checked to reach a given set of conclusions. The tree is encapsulated in an Ada package.

These two types of rule-based systems were tested with a small set of 20 rules that formed part of an aimpoint function. These systems were executed on a $15-\mathrm{MHz} 1750$ single-board computer (SBC), a 16-MHz 68020 SBC, and a Micro VAX 11 running Unix. As a baseline, an inference engine written in LISP and using the same rule set was on Symbolics workstation. The time from receipt of data to transmittal of the conclusions was measured. The results of these executions are given in Table 1. As expected, the 68020 SBC performed the best among all of the different computers and the decision tree was decidedly faster, by a factor or two or so. The difference in times for the Ada systems is due strictly to the hardware platform; since the same vendor supplied the different compilers and all of them are based on the same compiler technology.

TABLE 1. Rule-Based System Times.

\begin{tabular}{|c|c|c|c|}
\hline Computer & Time (ms) & Language & System \\
\hline $17450 \mathrm{~A}$ & 63 & Ada & $\begin{array}{l}\text { Inference } \\
\text { engine }\end{array}$ \\
\hline Symbolics & 29 & LISP & $\begin{array}{l}\text { Inference } \\
\text { engine }\end{array}$ \\
\hline Micro VAX II & 13 & Ada & $\begin{array}{l}\text { Inference } \\
\text { engine }\end{array}$ \\
\hline 68020 & 6 & Ada & $\begin{array}{l}\text { Inference } \\
\text { engine }\end{array}$ \\
\hline Micro VAX II & 5 & Ada & $\begin{array}{c}\text { Decision } \\
\text { tree }\end{array}$ \\
\hline 68020 & 2 & Ada & $\begin{array}{c}\text { Decision } \\
\text { tree }\end{array}$ \\
\hline
\end{tabular}

While a decision tree has an advantage in execution performance, it is at a disadvantage with regards to flexibility. If the rule set gets changed at all, a new decision tree must be generated, which then must be compiled and linked into the rest of the application software. An inference-engine-based system, while slower, is more flexible. The rule set can change without requiring any software to be rewritten, allowing the rule set to be handled like any other external data that is incoming to the system. Imagine a scenario where a rule set can be downloaded to a missile that is specific to the geographic location and intended mission. A decision-tree system would require all possible rule sets to be onboard, taking up memory when only one rule set is really needed. The choice of which type of system to use is dependent upon the application. A system that could combine the flexibility of an inference engine with the speed of a decision tree is most desirable. We are focusing our current effort on developing such a system. 
RULES CONDITION-TO-RULE LINK

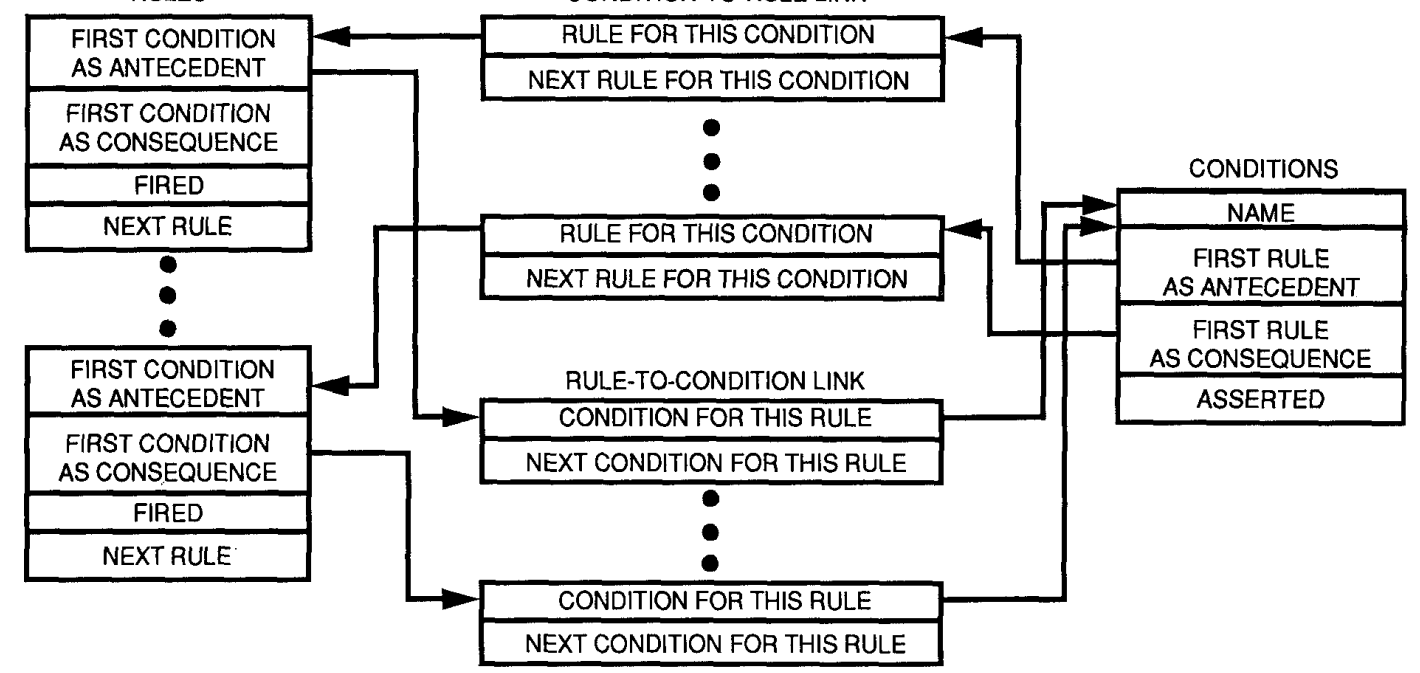

FIGURE 1. Data Structure.

The use of Ada has both advantages and disadvantages. Note from Figure 1 that the only consequences allowed are conditions (i.e., variables). When a rule fires, no actions can be initiated by the inference engine because Ada does not allow pointers to procedures or functions. We can work around this situation (sort of) by allowing only a predefined set of procedures that can be specified as consequences. Similar steps can be taken to handle functions as antecedents. Another way to work around the situation is to use a very small assembly language or $C$ program to do the calling through the pointer. Hopefully, this feature will make it through the Ada 9X revision process and be added to the Ada language. Additionally, Ada does not have an address operator (like C). This problem is minor and is easily solved.

\section{Hardware Assistance}

Until recently, implementation of rule-based systems could only be done using software. However, starting last year, a number of companies now offer chips that implement the reasoning process in hardware. The advantage is the same as that of doing floating-point operations in hardware versus doing those operations in software: speed. A hardware implementation is 20 to 2,900 times faster than software. Because a major concern in using rule-based systems in missile has been execution performance, these chips offer a significant breakthrough in the ability to use rule-based techniques. However, you can't get something for nothing. Adding another processor means increasing the power consumption, increasing the heat load, and taking up more real estate. Real estate is always at a premium-most tactical missiles have only between 100 to 200 cubic inches available for the computer system.
Most of the processors are built to handle fuzzy logic. Fuzzy logic is a superset of Boolean or binary logic, and was invented in the mid 1960 s by Dr. Zadeh, a professor a the University of California at Berkeley. Instead of just allowing true (1) or false (0) values, fuzzy logic allows the use of all the values in between ( 0 to 1$)$. This type of logic provides a way of handling uncertainty in information, which always exists to one degree or another. Because of these features, a fuzzy rule-based system has fewer rules than an equivalent system that uses binary logic. Current applications using fuzzy logic include elevator controls, subways, automatic transmissions, and autofocusing in cameras.

Four companies in the United States make fuzzy processors. Apt Instruments makes the CS/1, a programmable controller with the capability of chaining rules in real time. The $\mathrm{CS} / 1$ has a throughput of $4,000,000$ rules per second. Micro Devices makes a fuzzy set comparator, the MD1210, that can compare one input against eight stored patterns or eight inputs against one stored pattern. The Mircroelectronics Consortium of North Carolina makes a processor (unnamed) that can go through $58,000,000$ rules per second. This chip is a general-purpose fuzzy-logic inference engine. Togai InfraLogic makes the $\mathrm{FC} 110$, also a general-purpose fuzzy processor, which can evaluate 370,000 rules per second.

For the more traditional rule-based system, we've only been able to find one hardware system. Rex, a chip-and-board level system marketed by Richo Corporation and International Chip Corporation, is built on a custom microprocessor. The inference engine is cast in the chip. Rex can handle forward chaining, backward chaining, and a mixture of both. Rex has throughput of $1,670,000$ rules per second. 
Currently, we are just starting to look into these hardware systems. Next year, we plan on acquiring one of these (or equivalent) systems and investigating it in detail. At the end of next year, we will compare the performance of this system against a software-based system already developed for a particular application. Besides performance and suitability, two other issues need to be resolved. One of these issues is concerned with physical requirements, such as size, power, and heat. The other issue is concerned with the level of support available, such as tools to assist the user in developing the rule set for the processor.

We also plan to look at processors that provide direct support for Ada in a real-time environment. The missile-flight software lends itself to being implemented as a federation of cooperating tasks. However, tasking in Ada has a tendency to be too slow to be useful, leading developers to come up with their own way of handling multi-tasking. Hardware assistance in the tasking area would negate the need for developers to do this, saving development time. Two chips that show promise are the Intel $80960 \mathrm{MC}$ processor and the Lund University Tasking Support coprocessor. The Intel processor is built around a core designed to serve embedded applications. In addition to on-chip floating-point support and protection for code and date, this processor provides multi-tasking and multi-processing support. Specifically, the processor provides for task dispatching, preempting and rescheduling, and semaphores in hardware. The Lund chip is to be used as a coprocessor and was designed to implement all the elements of an Ada rendezvous in hardware. Delays, queue headers, and scheduling are all handled on the chip. Throughput performance was about 150 times faster than the software with a complex rendezvous taking less than 15 microseconds.

\section{Automated Tools}

Another aspect of the Intelligent Missile Project is to address two areas of software productivity. The first area is concerned with developing software that incorporates both rule-based systems and the standard algorithmic/control software. In current practice, this integration and checking is done manually and is time-consuming. The second area to be addressed is concerned with automating the requirements/ analysis phase of software development. Again, in current practice this is a manual process. We are currently working on the first area of concern; efforts on the second area will start next year.

Expert System Shells (ESS) provide for validating and verifying the rule set, and Computer Aided Software Engineering (CASE) tools provide for checking the analysis and design of traditional software. However, nothing exists that provides for any error checking across the respective domains. It is entirely possible that each portion of the software design, rule base and algorithmic/control of a system are individually correct but when these subsystems are integrated inconsistencies arise. These inconsistencies can happen because there is no central repository that contains information from both designs. This discrepancy could be resolved if there were a means of sharing the design data, and work was started on accomplishing just that. Besides evaluating the tools' capabilities in their respective domains, we also examined them to determine just how amenable these tools are to the sharing of data. Each tool was then ranked according to how well it scored in each of three categories: applicability, integrability, and supportability. Two CASE tools and one ESS scored high in all three categories. We purchased one tool for each domain and are now in the process of developing the software that will interface the two tools. Currently, each product's structures and verification mechanisms are being analyzed for commonality.

Future work in this area is concerned with the lack of tools to assist the software developer in the early stages of development. Reliable and error-free software depends upon a good design, which in turn depends upon a set of requirements. Errors in the requirements phase lead to design errors, which in turn lead to coding errors that result in undesired behavior in the software. The earlier in the life cycle an error is caught, the cheaper it is to correct it. The cost of correcting an error in the analysis phase is 100 times less than the cost of correcting an error when the software is operational.

The current method of determining software requirements is to manually read through several thick documents. This method is labor intensive, time consuming, and prone to error. The requirements are usually inconsistent and incomplete, though it takes some time to find this out. Our task is to automate the process of determining the software requirements, given the typical textual form of the requirements, and to determine the best way of presenting these requirements. Our approach is as follows: First, we will focus on analyzing the documentation for semantic content and determining what the relevant objects are. Object dependencies will be computed. Next, each individual object's behavior, including performance criteria (such as timing and accuracy) and the object's primary function(s) will be determined. With this information, a data or object flow diagram can be drawn. To show proof of concept, a requirements documentation package for an actual missile will be scanned in to a computer. The output will consist of one of the above, mentioned diagrams and a report of any inconsistencies or other errors that were found. This work is not without risk. Semantic analysis is a relatively new field strongly tied to natural language parsing. If the expected advances in this technology are not made, this work will not achieve its goals.

\section{Applications}

The purpose of the applications task is to test the developed methods in applications involving actual missiles. Three candidate systems were involved. The first system dealt with fusing the data from an RF seeker and and IR seeker. The second system was concerned with the use of a rule-based system for performing adaptive guidance in an air-to-surface missile. This rule-based system will take sensor and state 
information and select the optimum guide law from a set of guide laws. The third system involves a rule-based system that will act as a control executive to determine which is the best target. This system will integrate information from various sensors along with a priori information and missile kinematics. The latter two systems are candidates for the use of the hardware-based rule system mentioned earlier.

\section{IR-RF Fusion}

The intent of the IR-RF fusion effort was to use a rule-based system to fuse information from the two different seekers in order to obtain the best picture of a target. Instead of doing a one-time hand-over from one seeker to another, as has been done in the past, the rule system would constantly evaluate the data returned from each seeker. This system would determine which seeker's data should be used to indicate where the target is. A major problem developed in trying to come up with a rule set that could handle all possible scenarios. A rule set could be generated for any individual scenario, but no one rule set could be generated for all scenarios. This difficulty is a weakness in rule-based technology. If a situation arises that is not covered by the rule set, then the system does not know what to do. In this particular case, the only way to solve the problem would have been to include rule sets for every single scenario. This solution was not feasible because the possible scenarios were numbered in the tens of thousands. Therefore, no further work was done on the IR-RF fusion effort.

\section{$\underline{\text { Adaptive Guidance }}$}

The main objective of the adaptive guidance task is to improve missile guidance. Conventional signal-processing techniques are inadequate for dealing with low-probability-ofintercept waveforms. The instantaneous bandwidth and sensitivity of the receiver limit the number of pulses available for analysis of the waveform. The software designed to solve this problem is enormously complex. Even with structured development techniques, the knowledge required by a human expert to modify the software to account for threats is becoming prohibitive. The use of rule-based technology can alleviate these problems.

The frame rate for guidance is relatively high (20 to $100 \mathrm{~Hz}$ ). This high rate makes a demanding test for a rule-based application. Therefore, we planned on using a decision tree right from the start. We originally planned on using the decision-tree generator developed by the Intelligent Missile Project. However, there are several ESSs that can now generate decision trees in Ada. We have purchased one and are now using it to try candidate rule sets. The resulting system will be tested in a six-degree-of-freedom simulation. A successful demonstration will improve loss-of-track performance, simplify maintenance of tactical code, and decrease the transition time between guidance states.

\section{$\underline{\text { Target Selection }}$}

The problem faced by the target selector task is to develop effective ways to employ target-classification algorithms in Automatic Target Recognition (ATR) systems for autonomous missiles. Regardless of the technology employed, these algorithms essentially perform a mapping function by processing sensor data and assigning a label to it. The degree of target discrimination is a function of the sensor data, and in most cases, only partial target classifications are provided. The desired final level of information is inferred, but not directly produced. Consequently, we will use more than one classification algorithm, (i.e., a priori information and target detections) during an engagement, depending upon the situation.

The main objective of this work is to develop a rule-based system that will function as the control executive and issue the commit decision. To achieve this function, we will have to determine the points during a mission where the different classification algorithms should be used. Also, we will need to know how to incorporate a priori information into the ATR process and how to handle a large number of non-target contacts, such as decoys and chaff.

A conceptual framework for accomplishing this function follows. The key idea is that the sensor has the capability of developing and maintaining a ground-stabilized map of all detected contacts. These contacts would be processed by a categorizer that would label each contact as jammer, decoy, chaff, or valid target. The ATR hardware would classify the target contacts with respect to size, class, and combatant/noncombatant status. The control executive would take this information, along with any a priori data (formation map, known targets, priorities) and targeting instructions, and formulate a commit decision. This decision could be to go after a designated target or a specific type of target, to go after the target with the highest priority, or in the case where only noncombatants can be found, to go after no target and safe the missile.

Work has just started on developing the heuristics. We have identified 12 different types of data that the system will have to handle; much of these data associated have probabilities or certainty factors. We are also considering how best to handle conflict solution, i.e. where what is actually out there is not what was expected. 


\section{Conclusions}

Based on our experience gained during this project, Ada can be used to develop rule-based systems. However, with the advent of rule-based processors, work in Ada will be slowed down at least temporarily while we investigate this new technology. The new hardware technology has the potential to overcome a major obstacle to using rule-based systems for hard real-time applications.

If the right application is chosen, rule-based systems can find a home in missiles. While this systems are not feasible for pattern recognition, they can still be used in areas where combinatorial explosion does not exist, or where enough information is available that allows formulation of rules to cover the general case.

\section{$\underline{\text { References }}$}

1. Patrick Winston, Artificial Intelligence, 1984.

2. AI Expert, various issues.

3. Electronic Engineering Times, various issues.

4. NWC Technical Memo, in process.

5. C. Negoita and D. Ralescu, Simulation, Knowledge-Based Computing and Fuzzy Statistics. 